\title{
Blimp1 Suppresses Chx10 Expression in Differentiating Retinal Photoreceptor Precursors to Ensure Proper Photoreceptor Development
}

\author{
Kimiko Katoh, ${ }^{1,2,3}$ Yoshihiro Omori, ${ }^{1,2}$ Akishi Onishi, ${ }^{1,2}$ Shigeru Sato,,${ }^{1,4,5}$ Mineo Kondo, ${ }^{6}$ and Takahisa Furukawa ${ }^{1,2}$ \\ ${ }^{1}$ Department of Developmental Biology, Osaka Bioscience Institute, and ${ }^{2}$ JST, CREST, Suita, Osaka 565-0874, Japan, ${ }^{3}$ Graduate School of Biostudies, Kyoto \\ University, Yoshida-Honmachi, Sakyo-ku, Kyoto 606-8501, Japan, ${ }^{4}$ Department of Ophthalmology and ${ }^{5}$ Department of Visual Science, Osaka University \\ Graduate School of Medicine, Suita, Osaka 565-0871, Japan, and 'Department of Ophthalmology, Nagoya University Graduate School of Medicine, Showa- \\ ku, Nagoya 466-8550, Japan
}

The zinc finger transcription factor Blimp1 plays fundamentally important roles in many cell lineages and in the early development of several cell types, including B and T lymphocytes and germ cells. Although Blimp1 expression in developing retinal photoreceptor cells has been reported, its function remains unclear. We identified Blimp1 as a downstream factor of 0tx2, which plays an essential role in photoreceptor cell fate determination. To investigate Blimp1 function in the mouse retina, we ablated Blimp1 in the developing retina by conditional gene targeting. In the Blimp1 conditional knockout (CKO) retina, the number of photoreceptor cells was markedly reduced in the differentiated retina. We found that the numbers of both bipolar-like cells and proliferating retinal cells increased noticeably, with ectopic localizations in the postnatal developing retina. In contrast, a reduction of the number of photoreceptor precursors was observed during development. Forced expression of Blimp1 by in vivo electroporation suppressed bipolar cell genesis in the developing retina. Multiple genes involved in bipolar development, including Chx10, were upregulated in the Blimp1 CK0 retina. Furthermore, we showed that Blimp1 can bind to the $C h x 10$ enhancer and repress $C h x 10$ enhancer activity. These results suggest that Blimp1 plays a crucial role in photoreceptor development by repressing genes involved in bipolar cell fate specification and retinal cell proliferation in differentiating photoreceptor precursors.

\section{Introduction}

The vertebrate retina is comprised of five types of neurons: photoreceptor, horizontal, bipolar, amacrine, and ganglion cells, and one type of glial cell, Müller glia. These cell types arise from a multipotent progenitor cell population in a stage-specific manner (Cepko et al., 1996). We showed previously that the Otx2 transcription factor is essential for the cell fate determination of photoreceptor cells (Nishida et al., 2003; Sato et al., 2007). The Otx 2 conditional knockout (CKO) mouse retina showed a total absence of photoreceptors and a marked increase of amacrinelike cells, suggesting that the cell fate of photoreceptor precursors was converted to that of amacrine-like cells in the absence of Otx2. To reveal the transcriptional network in developing photo-

\footnotetext{
Received Feb. 12, 2010; revised March 17, 2010; accepted March 24, 2010.

This work was supported by Core Research for Evolutional Science and Technology of the Japan Science and Technology Agency; a grant for Molecular Brain Science, Grants-in-Aid for Scientific Research on Priority Areas, and a Grant-in-Aid for Scientific Research (B), Young Scientists (B), from the Ministry of Education, Culture, Sports and Technology of Japan; the Takeda Science Foundation; the Uehara Memorial Foundation; the Novartis Foundation; the Mochida Memorial Foundation for Medical and Pharmaceutical Research; the Naito Foundation; and a Grant-in-Aid for Scientific Research from the Japan Society for the Promotion of Science for Junior Scientists. We thank Dr. Kathryn Calame for Blimp1-flox mice; Dr. Shiming Chen for technical advice on ChIP; Dr. Michinori Saitou for Blimp1-mVenus mice; Drs. T. Koyasu, K. Miyata, and S. Ueno for ERG analysis; and M. Kadowaki, A. Tani, T. Tsujii, Y. Kawakami, A. Ishimaru, Y. Saioka, M. Joukan, K. Sone, and S. Kennedy for technical assistance.

Correspondence should be addressed to Takahisa Furukawa, Department of Developmental Biology, 0saka Bioscience Institute, 6-2-4 Furuedai, Suita, 0saka 565-0874, Japan. E-mail: furukawa@obi.or.jp.

D01:10.1523/JNEUROSCI.0771-10.2010

Copyright $\odot 2010$ the authors $\quad 0270-6474 / 10 / 306515-12 \$ 15.00 / 0$
}

receptor cells, we performed a genomewide expression profiling of the Otx2 CKO retina (Y. Omori, K. Katoh, S. Sato, and T. Furukawa, unpublished data). We identified the B lymphocyteinduced maturation protein 1 [Blimp1, also known as $P R$ domain containing $1(\operatorname{Prdm} 1)]$ gene as one of the downstream targets of Otx2. Blimp1 is a Prdm family transcription factor and was originally identified as a repressor of $\beta$-interferon gene expression (Keller and Maniatis, 1991). Blimp1 has been shown to function mainly as a transcriptional repressor. Blimp1 orchestrates development and homeostasis in a diverse variety of tissues through repression of cell-cycle progression and/or global change of gene expression (Shaffer et al., 2002; Ohinata et al., 2005; ShapiroShelef and Calame, 2005; Kallies and Nutt, 2007). Blimp1 functions as a master regulator of $\mathrm{B}$ lymphocyte terminal differentiation by repressing both cell proliferation and expression of a large number of genes critical for B lymphocyte identity (Turner et al., 1994). Blimp1 also specifies germ cell fate through silencing of the default somatic gene program and promotion of germ cell-related gene expression (Ohinata et al., 2005; Vincent et al., 2005). In T lymphocytes, Blimp1 governs T lymphocyte homeostasis by attenuating both proliferation and survival (Kallies et al., 2006; Martins et al., 2006). It was reported that Blimp1 is involved in specification of both neural crest cells and a subset of sensory neurons in zebrafish (Roy and $\mathrm{Ng}$, 2004). In the mouse retina, several studies reported that Blimp1 is expressed in the photoreceptor cell layer (Chang et al., 2002; Wilm and Solnica- 
Krezel, 2005; Hsiau et al., 2007). However, Blimp1 function in the developing retina remains elusive.

In the present study, to assess in vivo Blimp1 function in the retina, we generated Blimp1 CKO mice by crossing Blimp1 flox/flox mice (Shapiro-Shelef et al., 2003) with Dkk3-Cre mice (Sato et al., 2007). We report here that Blimp1 CKO mice showed a significant decrease of photoreceptor precursors and, in contrast, a marked increase of bipolar-like cells and proliferating retinal cells during development. Forced expression of Blimp1 leads to a specific reduction of developing bipolar cells. In addition, we demonstrated that Blimpl interacts with Chx10 enhancer regions and represses Chx10 transcription. These results suggest that Blimp1 ensures the proper development of photoreceptor cells through suppressing genes involved in bipolar cell fate specification and retinal cell proliferation in differentiating photoreceptor precursors.

\section{Materials and Methods}

Generation of Blimp1 CKO mouse. We mated a Blimp $1^{\text {flox }}$ (Shapiro-Shelef et al., 2003) mouse line with a Dkk3-Cre-BAC transgenic mouse line (Sato et al., 2007), which expresses Cre recombinase under control of the Dkk3 promoter. The Blimp1-mVenus (Ohinata et al., 2008), Crx-Cre (Nishida et al., 2003), and Crx-LacZ (Furukawa et al., 2002) transgenic mouse lines were described previously. All procedures conformed to the Statement for the Use of Animals in Ophthalmic and Vision Research of the Association for Research in Vision and Ophthalmology, and these procedures were approved by the Institutional Safety Committee on Recombinant DNA Experiments and the Animal Research Committee of Osaka Bioscience Institute. Mice were housed in a temperaturecontrolled room with a $12 \mathrm{~h}$ light/dark cycle. Fresh water and rodent diet were available at all times.

Histology and immunohistochemistry. Embryonic ages were determined as the time since the appearance of the vaginal plug [the first day was designated as embryonic day 0.5 (E0.5)]. Mouse embryos and eye cups were fixed in $4 \%$ paraformaldehyde in PBS for either $30 \mathrm{~min}$ to $3 \mathrm{~h}$ (for immunostaining) or overnight (for in situ hybridization). The samples were cryoprotected, embedded, frozen, and sectioned (20 $\mu \mathrm{m}$ thick). Slides were incubated with blocking solution ( $4 \%$ normal donkey serum and $0.5 \%$ Triton $\mathrm{X}-100$ in PBS) for $1 \mathrm{~h}$, and then with the primary antibodies for $4 \mathrm{~h}$ at room temperature. Slides were washed with PBS three times for $10 \mathrm{~min}$ each time and incubated with the secondary antibodies for $2 \mathrm{~h}$ at room temperature. The specimens were observed under a laser confocal microscope (LSM510; Carl Zeiss). We used the following primary antibodies: mouse monoclonal antibodies specific to $\beta$-galactosidase (1:500; Developmental Studies Hybridoma Bank), Crx (1:3000; Medical \& Biological Laboratories), calbindin D-28K (1:500; Sigma), Islet1 (1:100; Developmental Studies Hybridoma Bank), Pax6 (1:200; Developmental Studies Hybridoma Bank), proliferating cell nuclear antigen (PCNA; 1:500; Dako Cytomation), rhodopsin RET-P1 (1:5000; Sigma), and S-100 $\beta$ (1:1000; Sigma); rabbit polyclonal antibodies to active caspase 3 (1:100; Promega), Chx10 (1:100) (Koike et al., 2007), Ki-67 (1:100; Neomarkers), M-opsin (1:300; Millipore), phospho-histone H3 (1:100; Millipore), PKC $\alpha$ (1:10,000; Sigma), recoverin (1:1000; Millipore), rhodopsin (1:5000; LSL), S-opsin (1:1000; Millipore), and Sox9 (1:750; Millipore); rat monoclonal antibodies against Blimp1 (1:200; Santa Cruz Biotechnology) and green fluorescent protein (GFP; 1:1000; Nakalai); goat polyclonal antibodies to Bhlhb5 (1:200; Santa Cruz Biotechnology), Brn3b (1:100; Santa Cruz Biotechnology), and Otx2 (1:500; R\&D Systems); a guinea pig polyclonal antibody to Trb2 (1:50; MBL); and a sheep polyclonal antibody against bromodeoxyuridine (BrdU; 1:300; Fitzgerald). We used rhodamine-labeled peanut agglutinin (1:300; Vector Laboratories) and Alexa Fluor 488 phalloidin (1:3000; Invitrogen). For BrdU experiments, mice were injected intraperitoneally with $50 \mu \mathrm{g} / \mathrm{g}$ body weight of BrdU for $3 \mathrm{~h}$ (see Fig. $3 K, L$ ) or two days (supplemental Fig. $S 4 A, B$, available at www.jneurosci. org as supplemental material) (retinal tissues) before being killed. Terminal deoxynucleotidyl transferase-dUTP nick end labeling (TUNEL) assays were performed using the in situ Apoptosis Detection Kit (Takara) according to the manufacturer's instructions. The full-length cDNAs of Otx2 and Crx were used as templates to generate probes for in situ hybridization as described previously (Furukawa et al., 1997; Nishida et al., 2003). A Blimp1 cDNA fragment for an in situ probe was obtained by reverse transcriptase PCR.

Transfection and luciferase assay. Luciferase reporter plasmids ( $p$ GL3Chx10-A, $p$ GL3-Chx10-B, and $p$ GL3-Chx10-C) were generated by subcloning an $\sim 2.5 \mathrm{~kb}$ fragment from around $20 \mathrm{~kb}$ upstream (Chx10 region A; -20102 to $-17585 \mathrm{bp}$ ) (Kim et al., 2008), an $\sim 0.7 \mathrm{~kb}$ fragment from around $7 \mathrm{~kb}$ upstream (Chx10 region $\mathrm{B} ;-7851$ to $-7183 \mathrm{bp}$ ), or an $\sim 1.0$ $\mathrm{kb}$ fragment from around $13 \mathrm{~kb}$ downstream (Chx10 region C; +12470 to +13358 bp intron 3 ) of the mouse Chx10 gene into the $p$ GL3-promoter luciferase reporter vector (Promega). The mouse Blimp1/pcDNA3.1 expression vector was a gift from Dr. Kathryn Calame (Columbia University Medical Center, New York, NY). The Neuro2A neuroblastoma cell line was used for the luciferase assay. Transient transfection and measurement of luciferase activity were as described previously ( $\mathrm{Fu}-$ rukawa et al., 1997; Omori et al., 2001).

Microarray analysis. Mouse retinas were dissected at postnatal day 6 (P6). Total retinal RNA (5 $\mu \mathrm{g})$ was isolated using TRIzol reagent (Invitrogen) and converted to cDNA using the One-Cycle cDNA synthesis kit (Affymetrix) according to the manufacturer's instructions. Biotinlabeled cRNA was prepared using the in vitro transcription labeling kit and hybridized to the GeneChip mouse genome 4302.0 array (Affymetrix). Signal intensity was determined using GeneChip operating software version 1.4.

Quantitative real-time PCR and Northern blot analysis. Quantitative real-time PCR (Q-PCR) was performed using SYBR GreenER Q-PCR Super Mix (Invitrogen) and Thermal Cycler Dice Real Time System Single MRQ TP870 (Takara) according to the manufacturer's instructions. Quantification was performed by Thermal Cycler Dice Real Time System software version 2.0 (Takara). The primer sequences are listed in supplemental Table S3 (available at www.jneurosci.org as supplemental material). For the Northern blot analysis, a 364 bp (574-937 bp) fragment of Blimp1 cDNA was used as a radiolabeled probe.

Electroretinogram. Electroretinographic recordings were performed as described in detail previously (Koyasu et al., 2008). In brief, mice were dark adapted overnight, and then anesthetized with an intraperitoneal injection of ketamine and xylazine. Electroretinograms (ERGs) were picked up with a gold-wire loop electrode placed on the cornea. The mice were placed in a Ganzfeld dome and stimulated with stroboscopic stimuli ranging from -7.0 to $1.0 \log \mathrm{cd}-\mathrm{s} / \mathrm{m}^{2}$ to elicit the scotopic ERGs, and with five levels of stimuli ranging from -1.0 to $1.0 \mathrm{log} \mathrm{cd}-\mathrm{s} / \mathrm{m}^{2}$ for the photopic ERGs. The photopic ERGs were recorded on a rod-suppressing white background of $1.3 \log \mathrm{cd}-\mathrm{s} / \mathrm{m}^{2}$.

Chromatin immunoprecipitation. Chromatin immunoprecipitation (ChIP) was performed as described previously (Peng and Chen, 2005) using an anti-Blimp1 antibody (Santa Cruz Biotechnology). The immunoprecipitated chromatin DNA was analyzed by PCR using gene-specific primers (supplemental Table S3, available at www.jneurosci.org as supplemental material).

In vivo electroporation into the mouse retina. The in vivo electroporation into the mouse retinas was performed as described previously (Matsuda and Cepko, 2004). P0 mice were electroporated in vivo with $p C A G-G F P$ or pCAG-Blimp1. P6 retinas were dissected and examined by immunohistochemical analysis using the anti-Chx10 antibody.

Statistical analysis. Statistical significance was calculated with a Student's $t$ test. A value of $p<0.03$ was taken to be statistically significant. Data are presented as means $\pm \mathrm{SD}$.

\section{Results}

Blimp1 is expressed in photoreceptor precursors during development

We showed previously that Otx2 is essential for the cell fate determination of photoreceptors (Nishida et al., 2003). To identify factors that are critical for retinal development, we performed a microarray analysis of the control and the Otx2 

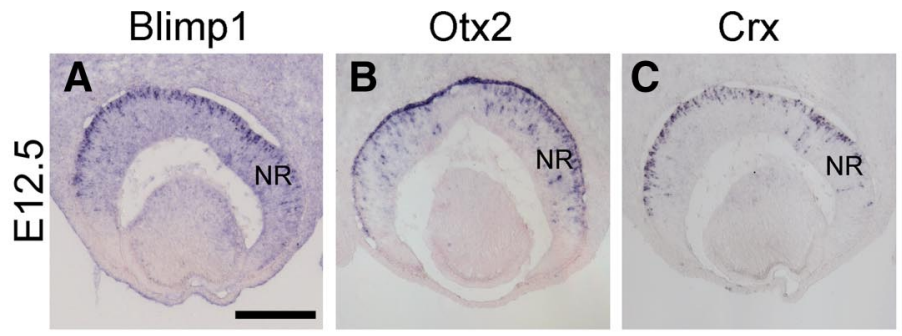

Blimp1-mVenus
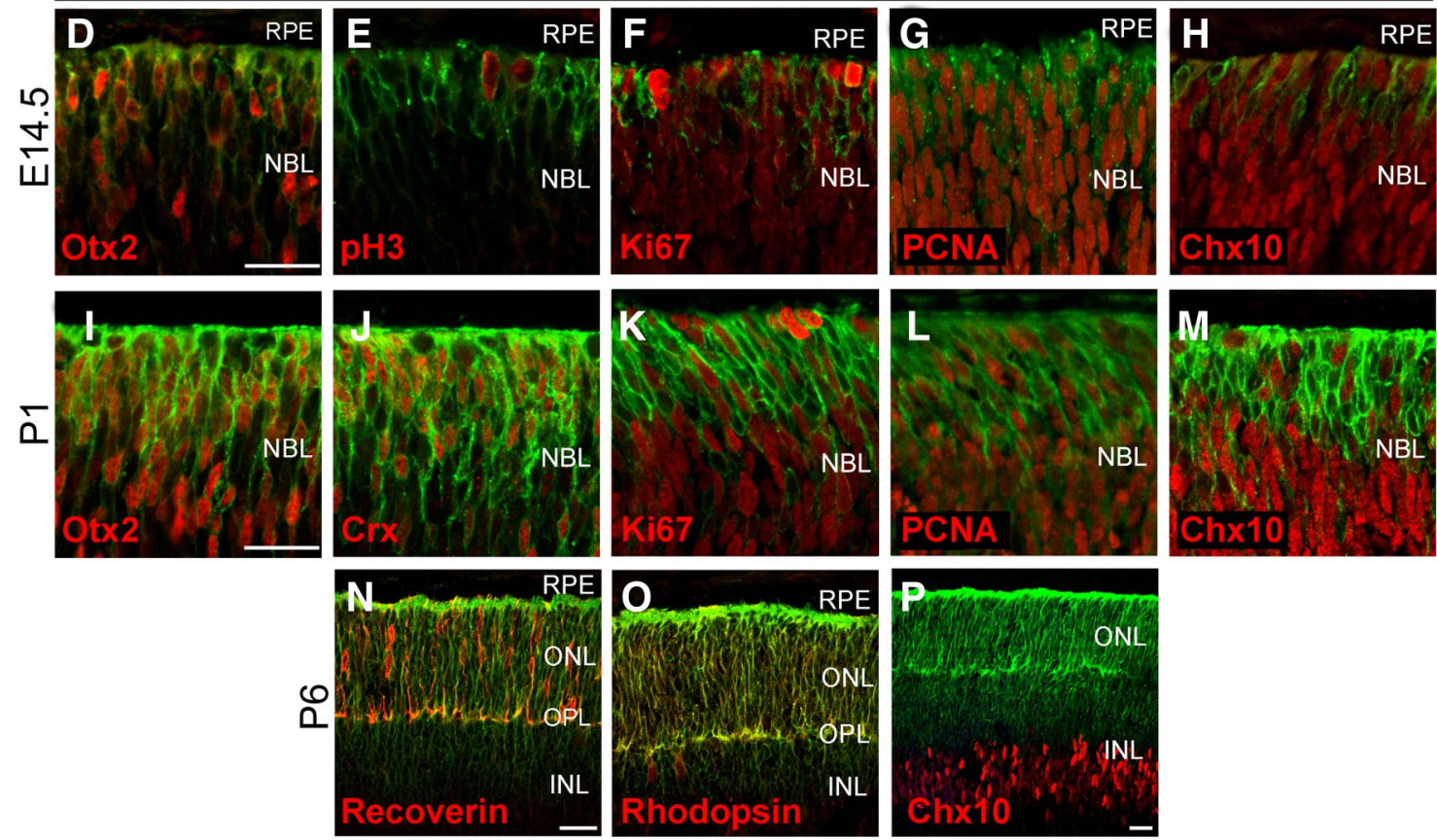

Figure 1. Blimp1 is expressed in photoreceptor precursor cells in the developing retina. $\boldsymbol{A}-\boldsymbol{C}$, Expression of Blimp $1(\boldsymbol{A}), 0$ tx $2(\boldsymbol{B})$, and $(r x(\boldsymbol{C}) \mathrm{mRNA}$ in the E12.5 mouse retina were detected by in situ hybridization. $\mathbf{D}-\boldsymbol{P}$, Immunostaining of the retina from a transgenic reporter mouse strain that expresses mVenus under the control of Blimp 1 regulatory elements (Blimp1-mVenus). Retinal sections were stained with anti-0tx2 $(\boldsymbol{D}, \boldsymbol{I})$, anti-pH3 $(\boldsymbol{E})$, anti-Ki67 $(\boldsymbol{F}, \boldsymbol{K})$, anti-PCNA $(\boldsymbol{G}, \boldsymbol{L})$, anti-Chx10 $(\boldsymbol{H}, \boldsymbol{M}, \boldsymbol{P})$, anti-Crx $(\boldsymbol{J})$, anti-Recoverin $(\boldsymbol{N})$, and anti-Rhodopsin $(\mathbf{O})$ antibodies at E14.5 $(\boldsymbol{D}-\boldsymbol{H})$, P1 $(I-M)$ and P6 (N-P). Scale bars: (in $\boldsymbol{A}) \boldsymbol{A}-\boldsymbol{C}, 200 \mu \mathrm{m} ;$ (in $\boldsymbol{D}, \boldsymbol{I}, \boldsymbol{N}, \boldsymbol{P}) \boldsymbol{D}-\boldsymbol{P}, 20 \mu \mathrm{m}$. NR, Neural retina; RPE, retinal pigment epithelium; NBL, neuroblastic layer.

CKO retinas (Omori, Katoh, Sato, and Furukawa, unpublished data). In this analysis, we identified that Blimp1 is almost absent in the Otx2 CKO retina at P1 and P12. We confirmed that Blimp1 was markedly downregulated in the Otx2 CKO retina by Q-PCR analysis ( $1.000 \pm 0.060$ in control; $0.012 \pm 0.003$ in Otx2 CKO; $p<0.03$ ).

It was reported previously that Blimp1 is expressed in the developing retina (Chang et al., 2002; Wilm and Solnica-Krezel, 2005; Hsiau et al., 2007). To determine the detailed expression pattern of Blimp1 in the developing retina, we performed in situ hybridization analysis of Blimp1 at several developmental stages. Blimp1 mRNA was first detected in the E11.5 retina (supplemental Fig. S1 A, available at www.jneurosci.org as supplemental material). Crx is an Otx2 family transcription factor that is positively regulated by Otx 2 and is essential for the terminal differentiation of photoreceptor cells (Furukawa et al., 1999). Similar to the Otx2 and $\mathrm{Crx}$ transcripts, a significant Blimp1 transcript signal was observed in the outer aspect of the neural retina corresponding to the prospective photoreceptor layer at E12.5 (Fig. 1 A-C). During embryonic and postnatal retinal development, Blimp1 expression was observed in the prospective photoreceptor layer from E15.5 to P3 (supplemental Fig. S1 $B-D$, available at www.jneurosci.org as supplemental material). After P6, the expression of Blimp1 decreased and became almost undetectable at the adult stage (supplemental Fig. S1 E, F, available at www.jneurosci.org as sup- plemental material). Consistent with the result by in situ hybridization, Northern blot analysis showed the expression of Blimp1 reaches its peak between P3 and P6 (supplemental Fig. S1G, available at www.jneurosci.org as supplemental material).

We then immunostained the developing retina from the transgenic reporter mouse strain which expresses membranetargeted Venus (mVenus) under control of the Blimp1 regulatory elements (Blimp1-mVenus mouse) (Ohinata et al., 2008). In the E14.5 retina, mVenus-positive cells overlapped with the Otx2 signal, a marker for photoreceptor precursors at this stage (Fig. $1 D)$. On the other hand, mVenus-positive cells rarely overlapped with the phospho-histone $\mathrm{H} 3$ ( $\mathrm{pH} 3$ ) signal (mitosis marker), the Ki67 signal (marker of proliferating cells), the PCNA signal (marker of proliferating cells), or the Chx10 signal (marker of progenitor in this stage) at E14.5 (Fig. $1 E-H)$. At P1, mVenuspositive cells overlapped with Otx2 and Crx signals, which are photoreceptor precursor markers (Fig. $1 I, J$ ). Conversely, the mVenus signal rarely colocalized with Ki67-, PCNA-, or Chx10positive cells at P1 (Fig. $1 K-M$ ). In the P6 retina, the cells expressing mVenus were co-stained with an anti-recoverin antibody or an anti-rhodopsin antibody, both of which are rod photoreceptor markers (Fig. $1 \mathrm{~N}, \mathrm{O}$ ). In contrast, mVenus-expressing cells rarely overlap with the Chx10 signal, which is a marker for immature and mature bipolar cells and proliferating progenitors (Fig. $1 H, M, P$ ). These results suggest that Blimp1 is predomi- 

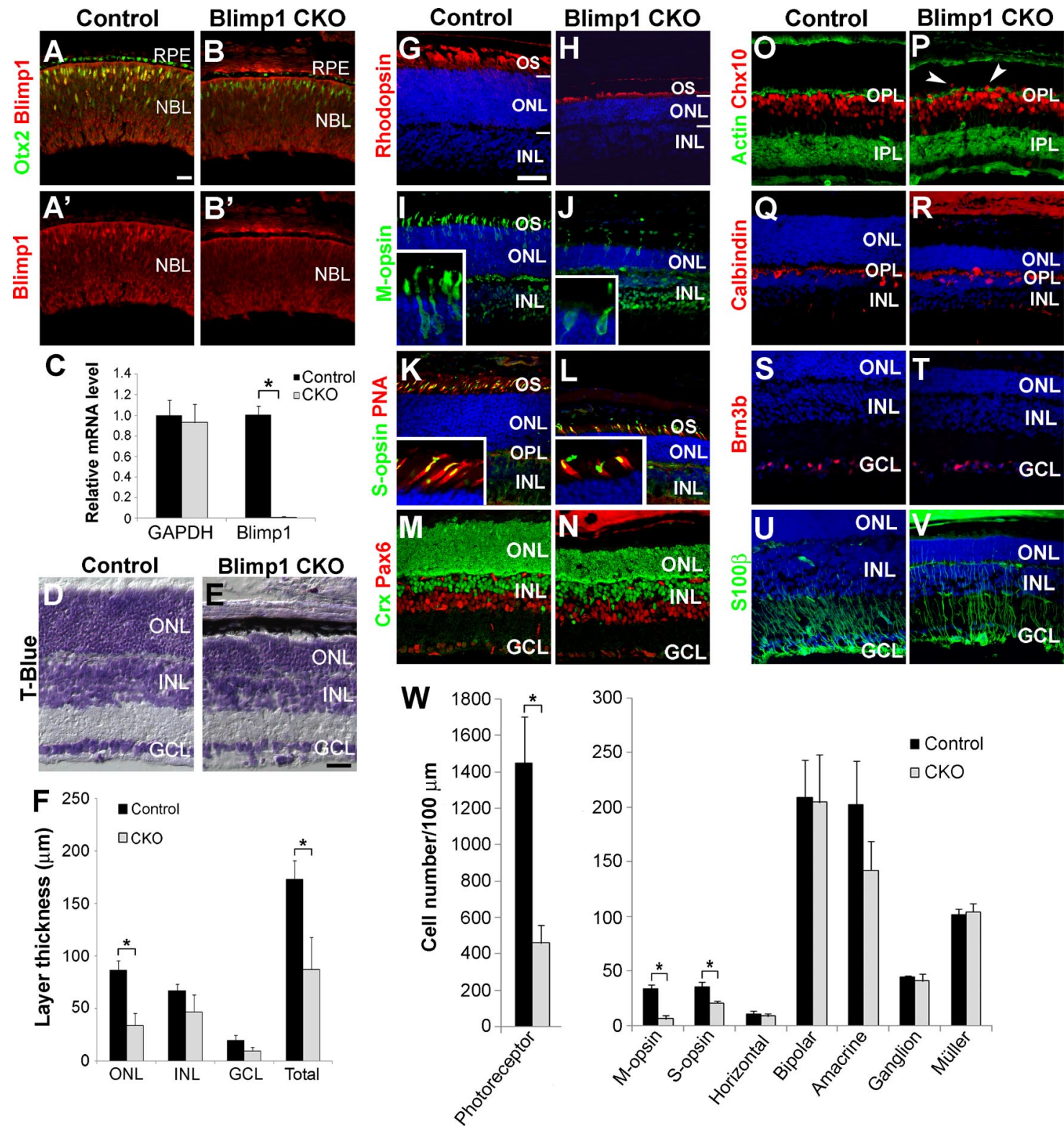

Figure 2. Targeted disruption of Blimp1 caused a specific reduction of photoreceptor cells in the retina. $\boldsymbol{A}-\boldsymbol{B}^{\prime}$, Retinal sections from the control $\left(\boldsymbol{A}, \boldsymbol{A}^{\prime}\right)$ and $B$ limp 1 CKO $\left(\boldsymbol{B}, \boldsymbol{B}^{\prime}\right)$ mice at E15.5 were stained with the anti-0tx2 (green) and anti-Blimp1 (red) antibodies. C, Expression levels of Blimp1 and GAPDH ( glyceraldehyde-3-phosphate dehydrogenase) were quantified by Q-PCR analysis in the control and Blimp 1 CKO retinas at P6 $(n=4) .{ }^{*} p<0.03$. D, E, Toluidine blue (T-Blue) staining of the control (D) and the Blimp1 CKO (E) retina at P14. $\boldsymbol{F}$, Thicknesses of GCL, INL, ONL, and total retina (total) were measured ( $n=15$ from 3 retinas). ${ }^{*} p<0.03$. G-V, P14 retinal sections from the control (left) and Blimp 1 CKO (right) mice were immunostained with cell-type-specific antibodies for rod photoreceptors (Rhodopsin; $\boldsymbol{G}, \boldsymbol{H}$ ), cone photoreceptors (M-opsin, $\boldsymbol{I}, \boldsymbol{J} ; \boldsymbol{S}$-opsin and PNA, $\boldsymbol{K}, \boldsymbol{L}$ ), photoreceptor and amacrine cells (Crx and Pax6; $\boldsymbol{M}, \boldsymbol{N}$ ), plexiform layers and bipolar cells (phalloidin, which stains actin in the $\mathrm{OPL}$, and $\mathrm{Chx10;} \mathbf{O}, \boldsymbol{P}$ ), horizontal cells (calbindin; $\boldsymbol{Q}, \boldsymbol{R})$, ganglion cells (Brn3b; $\boldsymbol{S}, \boldsymbol{T})$, and Müller glia $(S 100 \beta ; \boldsymbol{U}, \boldsymbol{V})$. The OPL stained with phalloidin was slightly irregular in the Blimp 1 CKO retina ( $\boldsymbol{P}$, arrowheads). Small boxes in $\boldsymbol{I}-\boldsymbol{L}$ show higher-magnification images. $\boldsymbol{W}$, Retinal cell type composition evaluated from section immunohistochemistry with various retina cell-specific markers. All data were represented as mean $\pm S D\left(n=15\right.$ from 3 retinas). ${ }^{*} p<0.03$. Scale bars: $50 \mu \mathrm{m}$. NR, Neural retina; RPE, retinal pigment epithelium; NBL, neuroblastic layer; 0 S, outer segment; IPL, inner plexiform layer.

nantly expressed in postmitotic photoreceptor precursors in the developing retina.

\section{Reduction of the number of photoreceptors in the Blimp1}

\section{CKO retina}

To investigate the in vivo function of Blimp1 in the developing retina, we ablated Blimp1 from the developing retina by conditional gene targeting. To accomplish this, we mated the Blimp $1^{\text {flox }}$ mouse line with the Dkk3-Cre transgenic mouse line in which Cre recombinase-mediated recombination occurs in almost all retinal progenitors (Sato et al., 2007). We obtained Blimp $1^{\text {flox/flox }}$; Dkk3-Cre ${ }^{+}$(Blimp1 CKO) mice by mating Blimp1 $1^{\text {flox/flox }}$ mice with Blimpl $1^{\text {flox/+}}$; Dkk3-Cre ${ }^{+}$mice. We analyzed the phenotypes and compared them to those of control mice with the genotype Blimp1 $1^{\text {flox/flox }}$; Dkk3-Cre ${ }^{-}$, which show no abnormal phenotype. Blimp1 CKO mice were viable and fertile. We confirmed a total loss of Blimp1 protein in the Blimp1 CKO retina at E15.5 by coimmunostaining with anti-Blimp 1 and anti-Otx 2 antibodies (Fig. $2 A-B^{\prime}$ ). We also observed an almost complete loss of Blimp1 mRNA expression in the Blimp1 CKO retina by Q-PCR analysis (Fig. 2C).

First, we examined the retinal tissue of the Blimp1 CKO and control mouse retinas at P14 by toluidine blue staining (Fig. $2 D, E)$. At this stage, retinogenesis is almost complete, and all 

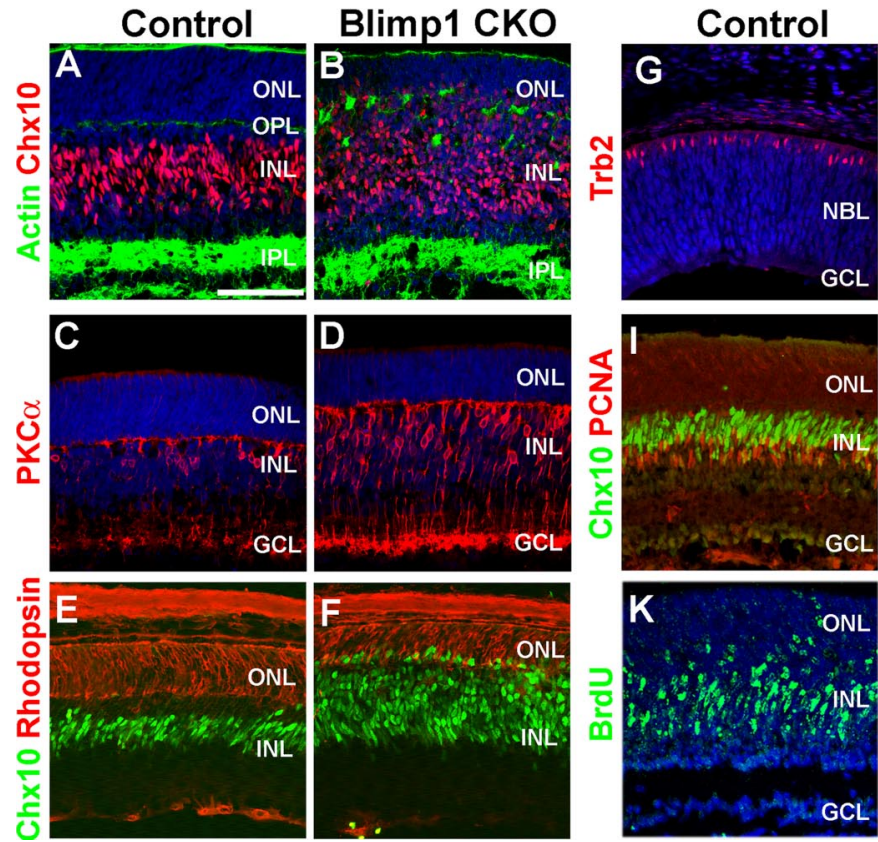

Figure 3. Cell composition is impaired in the developing Blimp1 CKO retina. $\boldsymbol{A}-\boldsymbol{D}$, The number of bipolar-like cells increased in the Blimp 1 CK0 retina $(\boldsymbol{B}, \boldsymbol{D})$ at P6 compared to the control $(\boldsymbol{A}, \boldsymbol{C})$ stained with the anti-Chx10 $(\boldsymbol{A}, \boldsymbol{B}$, red) and anti-PKC $\alpha(\boldsymbol{C}, \boldsymbol{D}$, red $)$ antibodies. The OPL is visualized by phalloidin staining $(\boldsymbol{A}, \boldsymbol{B}$, green), which stains actin filaments in the plexiform layers. $\boldsymbol{E}, \boldsymbol{F}$, Decreased number of photoreceptors in the Blimp1 CKO retina $(\boldsymbol{F})$ compared to the control $(\boldsymbol{E})$ at P6. Maturing photoreceptor precursors are stained with anti-rhodopsin antibody (red). $\mathbf{G}, \boldsymbol{H}$, Cone photoreceptor precursors decreased in the Blimp1 CKO at E14.5. Retinal sections from control $(\boldsymbol{G})$ and Blimp1 CKO $(\boldsymbol{H})$ mice were stained with the anti-Trb2 antibody (red), a marker for cone precursor in the embryonic retina. $I-L$, Increased numbers of proliferating cells were observed in the Blimp 1 CKO $(\boldsymbol{J}, \boldsymbol{L})$ compared to the control $(\boldsymbol{I}, \boldsymbol{K})$. Proliferating cells are stained with the anti-PCNA antibody $(\boldsymbol{I}, \boldsymbol{J}$, red) and $\operatorname{BrdU}(\boldsymbol{K}, \boldsymbol{L}$, green). Bipolar cells and proliferating retinal cells are visualized by the anti-Chx10 antibody (I, J, green). Scale bars: $50 \mu \mathrm{m}$. NBL, Neuroblastic layer.

three nuclear layers and the two plexiform layers are fully formed in both the control and Blimp1 CKO retinas (Fig. 2D-F). However, the thickness of the outer nuclear layer (ONL) is significantly thinner in the Blimp1 CKO retinas compared with that of the control retinas (Fig. $2 D-F$ ). In the mouse retina, $\sim 97 \%$ of photoreceptors are rod (Carter-Dawson and LaVail, 1979). The number of rod photoreceptors, determined by counting the number of 4', $6^{\prime}$-diamidino-2-phenylindole (DAPI)-positive nuclei in the ONL of the Blimp1 CKO retina at P14, was markedly decreased (Fig. $2 W$ ). Cone photoreceptors stained with the antiS-opsin and M-opsin antibodies also decreased (Fig. $2 I-L, W$ ). The length of outer segments of both rod and cone photoreceptors in the Blimp1 CKO retinas were significantly shorter than those of the control retinas (Fig. $2 G-L$ ). In contrast to the decrease of the number of photoreceptors, the numbers of the Chx10-positive bipolar cells, calbindin-positive horizontal cells, Pax6-positive amacrine cells, Brn3b-positive ganglion cells, and S100 $\beta$-positive Müller glial cells in the Blimp1 CKOs were unaffected at this stage, compared to those in the control retinas (Fig. $2 M-W)$. The outer plexiform layer (OPL) stained with phalloidin was slightly irregular in the Blimp1 CKO retinas (Fig. 2O,P).

Next, to evaluate the retinal physiological function in vivo, ERGs were recorded from 8-week-old Blimp1 CKO and control mice (supplemental Fig. S2, available at www.jneurosci.org as supplemental material). The scotopic ERG amplitudes of Blimp1 CKO mice were significantly smaller than those of the control mice, and the degree of amplitude reduction was similar for the aand b-waves (supplemental Fig. S2A,B, available at www.jneurosci. org as supplemental material). We found that the photopic ERG amplitudes of the Blimp1 CKO mice were significantly
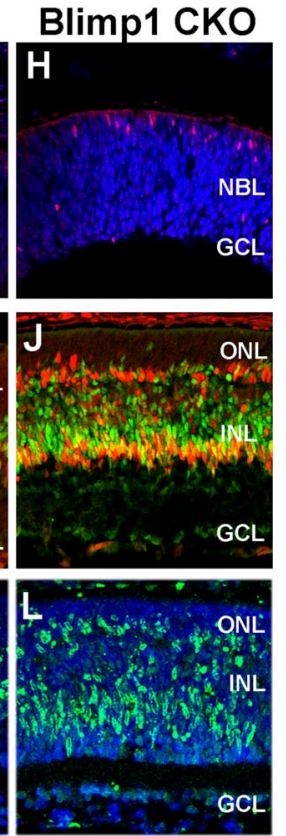

smaller than those of the control mice (supplemental Fig. S2C,D, available at www.jneurosci.org as supplemental material). These results suggest that the functional abnormality of the Blimp1 CKO retina lies mainly in the rod and cone photoreceptors.

\begin{abstract}
Abnormal cell composition in the developing Blimp1 CKO retina

We then examined retinal development of Blimp1 CKO retinas at E14.5, E15.5, P3, P6, and P9 and compared them to those of control retinas. We observed the most severe abnormality at P6 in the Bilmp1 CKO retina (Fig. $3 A-F, I-L$ ). In the normal retina, the production of bipolar cells reaches its peak at around P3 (Cepko et al., 1996). We found that the thickness from the inner nuclear layer (INL) up to the ONL was similar between the control $(112.6 \pm 8.2$ $\mu \mathrm{m})$ and the Blimp1 CKO retinas (104.1 \pm $11.1 \mu \mathrm{m}$ ) at P6 (Fig. 3A,B). However, ONL thickness of the Blimp1 CKO retina was significantly reduced $(47.4 \pm 5.8 \mu \mathrm{m}$ in control; $29.4 \pm 4.5 \mu \mathrm{m}$ in $\mathrm{CKO} ; p<$ 0.03 ) (Fig. $3 A, B$ ). Notably, we found that the number of Chx10-positive cells increased in the Blimp1 CKO retina, as did the dispersion of those Chx10-positive cells (number of $\mathrm{Chx} 10^{+}$cells $/ 100 \mu \mathrm{m}$, $146.2 \pm 31.8$ in control, $373.0 \pm 64.3$ in CKO; $p<0.03$ ) (Fig. $3 A, B$ ). We also ob-
\end{abstract} served an increased number of PKC $\alpha$-positive cells, which are rod bipolar cells (Fig. 3C,D). Chx10 is known to be expressed in both all bipolar cells and proliferating progenitor cells; therefore, we next coimmunostained with Chx10 and a proliferating cell marker, PCNA. Most of the ectopically localized Chx10-positive cells did not overlap with PCNA in the Blimp1 CKO retina (Fig. $3 I, J)$, suggesting that bipolar cells are overproduced in the developing Blimp1 CKO retina at P6. Similar to the P14 Blimp1 CKO retina, the number of differentiating rod photoreceptor precursors detected by the rhodopsin or recoverin signal was markedly decreased in the P6 Blimp1 CKO retina (number of recoverin cells/100 $\mu \mathrm{m}, 84.8 \pm 15.6$ in control, $47.0 \pm 3.8$ in $\mathrm{CKO} ; p<$ 0.03 ) (Fig. $3 E, F$ ).

Furthermore, we also found a remarkable increase of PCNApositive proliferating cells in the Blimp1 CKO at P6 (number of PCNA ${ }^{+}$cells $/ 100 \mu \mathrm{m}, 48.0 \pm 5.7$ in control, $103.6 \pm 7.9$ in CKO; $p<0.03$ ) (Fig. $3 I, J)$. Consistent with this, after BrdU pulse labeling for $3 \mathrm{~h}$ in the $\mathrm{P} 6$ retina, we found increased numbers of BrdU-positive cells in the Blimp1 CKO retina (Fig. $3 K, L$ ). All these observations suggest that lack of Blimp1 in the developing retina causes an abnormal increase of Chx10-positive cells and proliferating cells.

To confirm that the loss of Blimp1 in photoreceptor precursors causes these abnormal retinal cell compositions, we generated Blimp1 flox/flox; Crx-Cre ${ }^{+}$(Blimp1 Crx-Cre CKO) mice. Crx-Cre mice specifically express Cre in photoreceptor precursors under the control of the Crx promoter (Furukawa et al., 2002). We observed reduced numbers of photoreceptor precursors and excess bipolarlike and proliferating cells in the Blimp1 Crx-Cre CKO retina at P6 (supplemental Fig. S3A-H, available at www.jneurosci.org as sup- 
plemental material), similar to the Blimp1 $\mathrm{CKO}$ retina. The retinal phenotype of Blimp1 Crx-Cre CKO is similar to but less severe than that of Blimp1 Dkk3-Cre CKO. This is likely because of Blimp1 expression beginning as early as $C r x$ expression and inactivation of Blimp1 in Crx-Cre mice occurring slightly later than in Dkk3-Cre mice.

What cells are these excess bipolar-like cells and proliferating cells in the Blimp1 $\mathrm{CKO}$ retina derived from? One possibility is that these cells are autonomously derived from photoreceptor precursors in which Bimp1 is inactivated. The other possibility is that the deficiency of Blimp1 in photoreceptor precursors secondarily causes abnormal production of bipolarlike cells and/or proliferating cells from cells other than photoreceptor precursor cells.

To test these possibilities, we used the Crx-LacZ transgenic mouse line in which $\beta$-gal, under the control of the $2 \mathrm{~kb}-\mathrm{Cr} x$ promoter, specifically labels photoreceptor precursor linage (Fig. 4A) (Furukawa et al., 2002). We generated Blimp $1^{\text {flox/flox }}$;


lyzed their retinas by immunostaining at $\mathrm{P} 1, \mathrm{P} 4$, and $\mathrm{P} 6$. In the control retina (Blimp1 $1^{\text {flox/flox }}$; Dkk3-Cre ${ }^{-}$; Crx-LacZ ${ }^{+}$), most of the $\beta$-gal-positive cells are negative for the Chx10 signal (Fig. $4 B, F, J$ ). We found that $\beta$-gal and Chx10 doublepositive cells increased in the Blimp1 CKO retina (number of $\beta$-gal ${ }^{+} \mathrm{Chx} 10^{+}$cells/ $100 \mu \mathrm{m}$ at $\mathrm{P} 4,2.6 \pm 1.7$ in control, $40.6 \pm$

10.1 in $\mathrm{CKO} ; p<0.03$ ) (Fig. $4 B, C, F, G, J, K$ ). Furthermore, $\beta$-gal and Ki67 double-positive cells also increased in the Blimp1 CKO retina (number of $\beta$-gal ${ }^{+} \mathrm{Ki}^{+} 7^{+}$cells $/ 100 \mu \mathrm{m}$ at $\mathrm{P} 4,9.6 \pm 4.7$ in control, $47.1 \pm 18.3$ in $\mathrm{CKO} ; p<0.03$ ) (Fig. $4 D, E, H, I, L, M)$. These results suggest that Blimp1-deficient photoreceptor precursors were converted to bipolar-like cells or proliferation marker-positive cells autonomously in the Blimp1 Dkk3-Cre $\mathrm{CKO}$ retina. In contrast, most rhodopsin-positive cells, which correspond to more mature photoreceptors than Crx-LacZpositive cells, rarely overlap with the Chx10 signal either in the Blimp1 CKO or control retinas (Fig. $3 E, F$ ).

It should be noted that we observed a significant number of Chx10-positive cells in the Blimp1 CKO retina at P6; however, we found no significant change in mature bipolar cell numbers between the control and Blimp1 CKO retinas at P14. One possible mechanism underlying this phenotype is the elimination of bipolar cells by apoptotic cell death after P6 in the Blimp1 CKO retina. To test this possibility, we examined apoptotic cell death by active caspase 3 staining and TUNEL assay in the Blimp1 CKO and the control retinas (Fig. 5A-M). We stained apoptotic cells using the anti-active caspase 3 antibody and counted the number of apoptotic cells in the retina at E15.5, E17.5, P1, P3, P6, P9, and P14. At all stages examined, we found the number of active caspase3positive cells significantly increased (Fig. $5 K$ ). Consistent with this, TUNEL-positive cells also significantly increased in the Blimp1 CKO retina (Fig. 5M). In addition, we separately counted the apoptotic cell numbers in the ONL, INL, and ganglion cell layer (GCL) at P6 and P9. We found that apoptotic cells increased in the ONL as well as in the INL of the Blimp1 CKO retina (Fig. $5 L$ ). These results suggest that a subset of aberrant photoreceptors died of apoptosis in early development, and that overproduced bipolar-like cells and proliferating cells in the Blimp1 CKO retina were eliminated by apoptotic cell death before maturation of bipolar cells. We also observed a significant increase of apoptotic cells in the early retina (Fig, $5 K$ ). Since rod photoreceptor production reaches its peak around P0 (Cepko et al., 1996), the increase of apoptotic cell death in the early Blimp1 CKO retina is likely caused by the death of aberrant rod precursors.

\section{Decrease of cone photoreceptors in the early Blimp1 CKO retina}

We observed a marked reduction of photoreceptor precursors in the Blimp1 CKO retina at P6. Most photoreceptors generated at this stage are rods. In contrast, the majority of cone photoreceptors are generated during the first wave of retinogenesis during embryonic stages (Cepko, 1996). To investigate whether Blimp1 function affects cone development, we examined the Blimp1 $\mathrm{CKO}$ retina at E14.5. We stained cone precursors in the embryonic retina using the anti-Trb2 antibody. We found that the number of Trb2-positive cells significantly decreased in the Blimp1 CKO retina at E14.5 (number of Trb2 ${ }^{+}$cells/100 $\mu \mathrm{m}$, $14.7 \pm 2.6$ in control, $7.4 \pm 0.4$ in $\mathrm{CKO} ; p<0.03$ ) (Fig. $3 G, H$ ). 

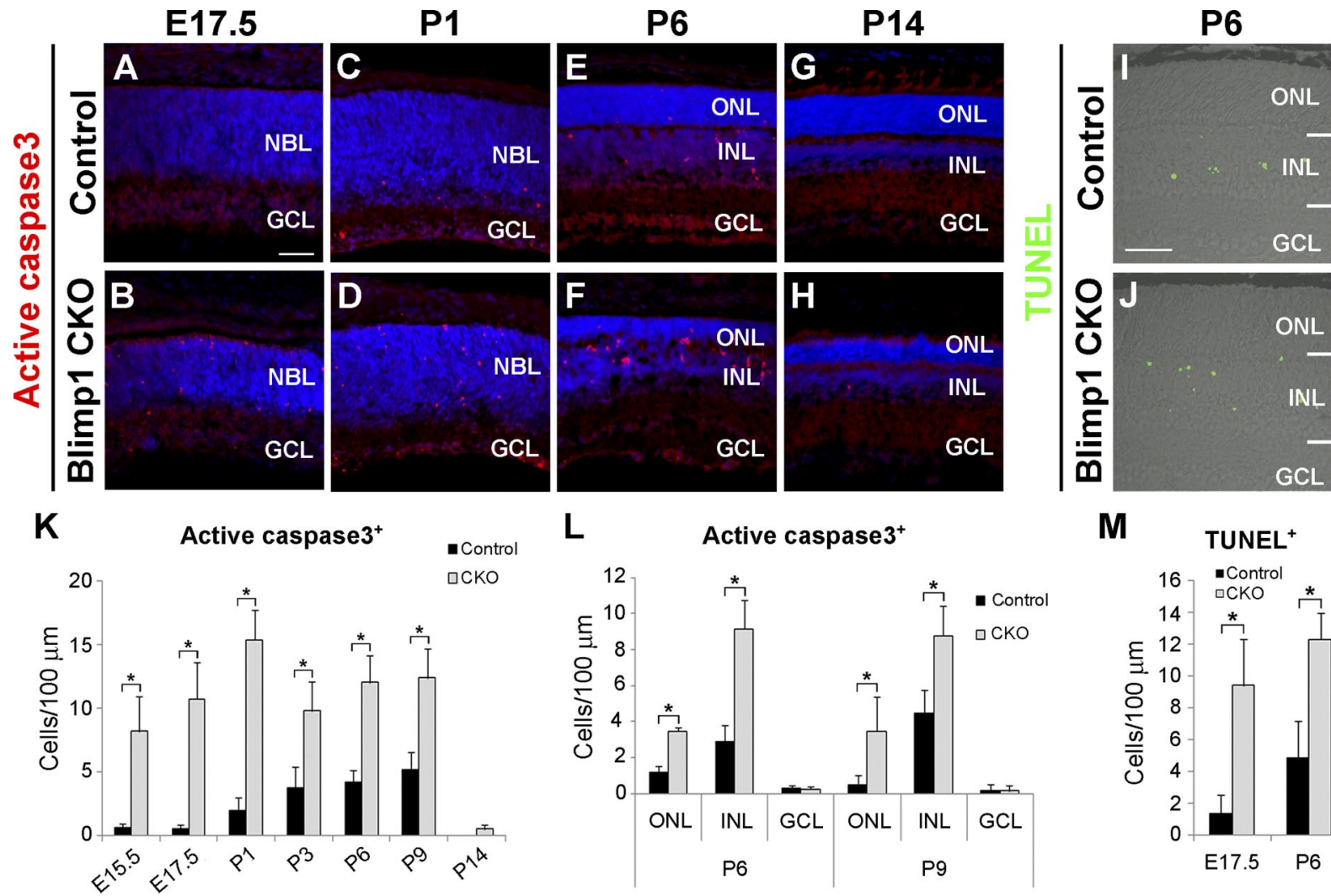

$\mathbf{L}$

Active caspase $3^{+}$

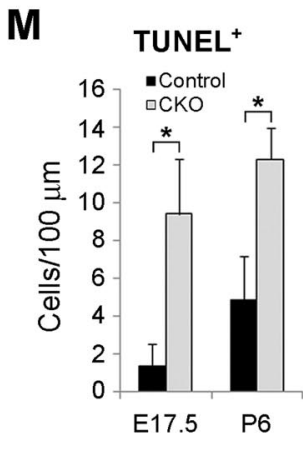

Figure 5. Significant increase of retinal cell death in the developing Blimp 1 CKO retina. $\boldsymbol{A}-\boldsymbol{H}$, Apoptotic cells were stained with anti-active caspase3 (red signal) in the control $(\boldsymbol{A}, \boldsymbol{C}, \boldsymbol{E}, \boldsymbol{G})$ and $B$ limp 1 CKO (B, $\boldsymbol{D}, \boldsymbol{F}, \boldsymbol{H})$ retinas atE17.5 $(\boldsymbol{A}, \boldsymbol{B}), \mathrm{P} 1(\boldsymbol{C}, \boldsymbol{D}), \mathrm{P} 6(\boldsymbol{E}, \boldsymbol{F})$, and P14 $(\boldsymbol{G}, \boldsymbol{H})$. Nuclei were stained by DAPI (blue signal). $\boldsymbol{I}, \boldsymbol{J}$, TUNEL staining of P6 retinas from control $(\boldsymbol{I})$ and Blimp1 $\mathrm{CKO}(\boldsymbol{J})$ mice. $\boldsymbol{K}-\boldsymbol{M}$, Cell numbers of active caspase3-positive cells $(\boldsymbol{K}, \boldsymbol{L})$ and TUNEL-positive cells $(\boldsymbol{M})$ in the control and Blimp1 CKO retinas were counted. ${ }^{*} p<0.03$. Scale bars: $50 \mu \mathrm{m}$. NBL, neuroblastic layer.

This result in the embryonic retina is consistent with a reduction of mature cones at later stages (Fig. $2 I-L, W$ ). In addition, we examined whether Blimp1 deficiency affects embryonic retinal cell fate choice by immunostaining using cell proliferation and cell type-specific markers. We observed that the numbers of BrdU- and $\mathrm{pH} 3$-positive proliferating cells, Brn3b-positive ganglion precursors, Bhlhb5-positive amacrine precursors, and Islet1-positive cells (horizontal, amacrine, ganglion precursors) were unaltered between the wild-type and Blimp1 CKO retinas (supplemental Fig. S4A$J, M-Q$, available at www.jneurosci.org as supplemental material). In contrast, the apoptotic cells were significantly increased in the Blimp1 CKO retina at E14.5 (supplemental Fig. S4 $K, L, R$, available at www.jneurosci.org as supplemental material). We also observed that Trb2 and Chx10 double-positive cells significantly increased in the Blimp1 CKO retina at E15.5 (supplemental Fig. S5 A, B, available at www.jneurosci.org as supplemental material). These results show that Blimp1 deficiency affects cone photoreceptor development. The aberrant cone precursors in the Blimp1 CKO retina appear to express Chx10 and die at this stage.

\section{Gene expression profiles of the Blimp1 CKO retina}

To investigate the molecular mechanisms underlying the histological phenotype of the Blimp1 CKO retina at $\mathrm{P} 6$, we analyzed a gene expression profile of the Blimp1 CKO retina using a microarray containing 45,101 probe sets covering $>34,000$ genes. We identified 222 downregulated probe sets (signal log ratio, less than or equal to -1.0 ; signal intensity, $\geq 100$ ) and 218 upregulated probe sets (signal $\log$ ratio, $\geq 1.0$; signal intensity, $\geq 100$ ) in the Blimp1 CKO retina, compared with the control retina (the lists of top 20 probes in each group are shown in supplemental
Tables S1, S2, available at www.jneurosci.org as supplemental material). Consistent with the results of immunohistochemical analysis, we observed several photoreceptor-specific genes (e.g., Arr3, Pde6h, and Rhbdf2) in these downregulated genes in the Blimp1 CKO retina. We also identified several bipolar cell-related genes (e.g., Scgn, $V s x 1$, and Cabp5) in the upregulated genes in the Blimp1 CKO retina. To confirm the differential expression of genes involved in bipolar and photoreceptor development in the Blimp1 CKO retina, we selected 11 genes involved in bipolar cell development and 10 genes involved in photoreceptor cell development, and assayed the expression levels of these genes in the Blimp1 CKO retina at P6 by Q-PCR analysis. Similar to the result of the microarray analysis, we found that the expression of several genes involved in bipolar development (Chx10, Vsx1, Lhx3, Lhx4, Prox1, Islet1, and Scgn) were notably upregulated (twofold to fivefold) in the Blimp1 CKO retina at P6 (Fig. 6A). In contrast, several genes involved in photoreceptor development [ $\mathrm{Cr} x, \mathrm{Nrl}$ (neural retina leucine zipper), Rbp3, NeuroD1, Thrb, Arr3, and $\mathrm{Sag}$ ] were significantly downregulated (by 50 to $80 \%$ from control) in the Blimp1 CKO retina (Fig. 6B). These results suggest that loss of Blimp1 in the developing photoreceptors leads to a downregulation of photoreceptor-related gene expression and to an upregulation of bipolar cell-related gene expression.

\section{Blimp1 can directly downregulate $C h x 10$ transcription}

What is the molecular mechanism underlying the overproduction of Chx10-positive cells and proliferating cells in the Blimp1 $\mathrm{CKO}$ retina? Ectopic expression of Chx10 has been shown to increase the number of bipolar-like cells and reduce the number of photoreceptors (Livne-Bar et al., 2006). This phenotype is par- 
tially similar to our observed phenotype in the developing Blimp1 CKO retina. In fact, we observed an increased number of Chx10-positive cells in the Blimp1 CKO retina. Blimp1 has been known to direct gene regulation during cell differentiation through the recruitment of interacting factors to specific sites (Gyory et al., 2004). Based on these observations, we focused on $C h \times 10$ as one of the putative targets of the Blimp1 transcription factor in the developing retina. We hypothesized that Blimp1 might suppress Chx10 expression in the photoreceptor precursors and prevent their cell fate change toward the bipolar cells.

To examine whether or not Blimp1 can directly suppress Chx10 enhancer activity, we performed a luciferase reporter assay using the Chx10 enhancer and the Blimp1 expression vector (Fig. 6C,D). A previous study showed that a $2.4 \mathrm{~kb}$ region upstream of the mouse Chx10 locus was sufficient to drive reporter expression in bipolar cells and proliferating progenitor cells in transgenic mouse lines (Rowan and Cepko, 2005) (Fig. 6C, region A). We identified at least two Blimp1-binding consensus sequences AGNGAAAG (Kuo and Calame, 2004; Ancelin et al., 2006) in this region, called region $A$. In addition, we searched other possible Blimp1-binding consensus sequences in the $C h \times 10$ locus between -10 $\mathrm{kb}$ and $+26 \mathrm{~kb}$, and identified two more regions (regions $\mathrm{B}$ and $\mathrm{C}$ ) containing at least two (region B) and four (region C) Blimp1binding consensus sequences GGGAAAG or AGNGAAAG, respectively. Region $B$ is located $\sim 7 \mathrm{~kb}$ upstream of the $C h \times 10$ gene, and region $\mathrm{C}$ is localized $\sim 12 \mathrm{~kb}$ downstream of the transcriptional starting site of the Chx10 gene. All of these regions are relatively well conserved among mammalian Chx10 genes.

To test whether or not Blimp1 is able to regulate transcription through these regions, we performed a luciferase reporter assay by transfecting both a Blimp1 expression plasmid and a luciferase reporter vector containing each of the three candidate regulatory regions of the Chxl0 gene, separately. We observed a significant dosedependent repression of the reporter gene by Blimp1 in all three constructs that we tested (Fig. 6D). To confirm the binding of Blimp1 to these regions in vivo, we performed a ChIP assay using an anti-Blimp1 antibody on P3 mouse retinal tissues. We found that Blimp1 specifically immunoprecipitated selected sequences in regions A and B; however, we did not detect a significantly amplified band in the ChIP assay for region $\mathrm{C}$ (Fig. $6 E$ ). These results suggest that Blimp1 is a direct upstream regulator of Chx10 via binding to the consensus sequences in the Chx10 upstream region.
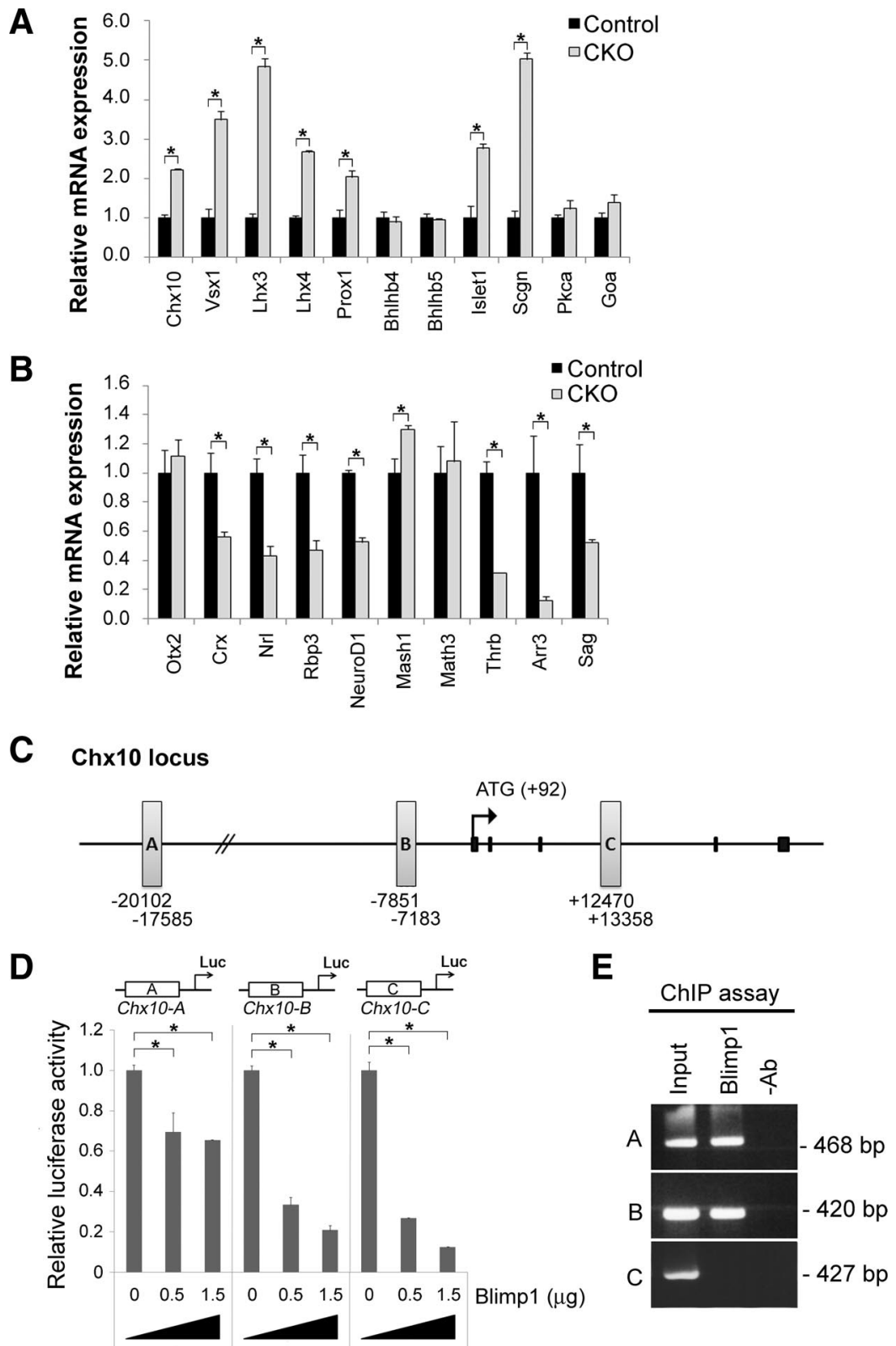

Figure 6. Gene expression profiling of the Blimp1 CKO retina by Q-PCR and direct regulation of Chx10 promoter by Blimp1. $\boldsymbol{A}, \boldsymbol{B}$, Expression levels of selected genes involved in bipolar $(\boldsymbol{A})$ and photoreceptor $(\boldsymbol{B})$ development were analyzed by Q-PCR. Expression levels of genes were normalized to the expression level of a housekeeping gene, GAPDH. Several genes involved in bipolar cell development were upregulated $(\boldsymbol{A})$ in the Blimp1 CKO retina. Several genes involved in photoreceptor development were downregulated in the Blimp1 CKO retina $(\boldsymbol{B})$. The mean value of each control was set as 1.0. Error bars show the SD $(n=4$ from 7 retinas). ${ }^{*} p<0.03$. C, Schematic diagram of three potential Blimp1-binding regions (shown as $A, B$, and C) found on $5^{\prime}$ upstream (regions $A$ and $B$ ) and downstream (region C) of the transcriptional start site (arrow) of the mouse Chx10 gene. $D$, Dose-dependent repression by Blimp1 of three possible Chx10 regulatory regions. Neuro2A cells were transfected with reporter plasmids containing region $A$, $B$, or $C$ along with various amounts of the Blimp 1 expression plasmid. The results were normalized against the Renilla luciferase activities obtained from the $p R L-T K$ plasmid as an internal control. Error bars show the SD $(n \geq 3)$. ${ }^{*} p<0.03$. E, Interaction of endogenous Blimp1 with genomic DNA of the Chx 10 locus by ChIP assay. Cell extracts (Input) from isolated $\mathrm{P} 3$ retinas were immunoprecipitated with (Blimp1) or without $(-\mathrm{Ab})$ the anti-Blimp1 antibody. Three putative Blimp1binding regions (regions $\mathrm{A}-\mathrm{C}$ ) were amplified by $\mathrm{PCR}$.

Overexpression of Blimp1 by electroporation inhibits bipolar genesis in the developing retina

To test whether Blimp1 is sufficient to suppress bipolar cell development, likely by suppressing Chx10 expression, we forced the expression of Blimp1 in the developing mouse retina by in vivo electroporation (Fig. 7). We electroporated both the Blimp1 ex- 


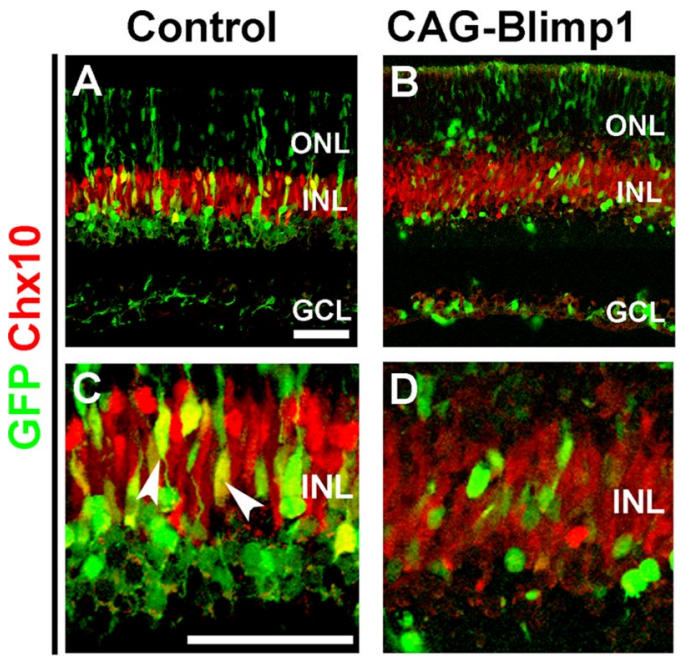

Figure 7. Overexpression of Blimp 1 suppresses bipolar cell genesis in the developing mouse retina. $\boldsymbol{A}-\boldsymbol{D}$, Immunohistochemical analysis of $\mathrm{P} 6$ retinas electroporated in vivo at $\mathrm{P0}$ with CAG-GFP $(\boldsymbol{A}, \boldsymbol{C})$ and CAG-Blimp1 $(\boldsymbol{B}, \boldsymbol{D})$ vectors. Bipolar cells were stained with the anti-Chx10 antibody (red). $\boldsymbol{C}, \boldsymbol{D}$, Higher-magnification images focused on the INL. White arrowheads point to Chx10-expressing electroporated cells. Scale bars: $50 \mu \mathrm{m}$.

pression vector and a GFP expression vector together, or a GFP expression vector alone as a control into P0 mouse retinas. At P6, when the most significant phenotype was observed in the Blimp1 $\mathrm{CKO}$ retina, transfected retinas were immunostained with the anti-Chx10 antibody and analyzed by confocal microscopy. Identification of cell types was determined by the characteristic morphologies and locations of cells. The majority of electroporated cells at $\mathrm{P} 0$ are rod precursors, which natively express Blimp1. We actually observed that the number of GFP-positive cells in the ONL was not significantly altered between the Blimp1 expression vector-electroporated $(50.5 \pm 9.2 \%)$ and control retinas $(53.5 \pm 5.0 \%$ ) (Fig. $7 A, B)$. In contrast, the Chx10 and GFP double-positive cells were markedly decreased in the Blimp1transfected cells $(20.7 \pm 3.8 \%$ in control, $6.1 \pm 5.2 \%$ in CAGBlimp $1 ; p<0.03$ ) (Fig. $7 A-D$ ). These results suggest that Blimp1 repressed bipolar cell development but not photoreceptor cell development. Consistent with the results from the loss of function experiments of Blimp1, forced expression of Blimp1 inhibited bipolar cell development in the developing retina.

\section{Discussion \\ Regulation of Blimp1 expression in differentiating photoreceptor precursor cells}

In the present study, we investigated the expression and function of the Blimp1 transcription factor in the developing retina. To explore Blimp1 expression in detail, the Blimp1-mVenus transgenic mouse line has been used (Ohinata et al., 2008). By taking advantage of the Blimp1-mVenus transgenic line, we found that Blimp1 is expressed mostly in postmitotic photoreceptor precursors, suggesting that Blimp1 may play a significant role downstream of the Otx2 transcription factor in photoreceptor precursor differentiation. In the current study, we showed Blimp1 expression in the embryonic retina begins as early as E11.5, when Otx2 begins to be expressed as well (Nishida et al., 2003); however, Otx2 expression was unaltered in the Blimp1 CKO retina. These results suggest that Blimp1 expression is regulated by Otx2, but Otx2 expression is not regulated by Blimp1. Unlike Otx2, whose expression is known to begin in the late $S$ to early G2 phase of the last cell cycle in retinal progenitor cells (Trimarchi et al., 2008a,b), our study showed that Blimp1 expression was mainly observed in postmitotic photoreceptor precursor cells. Only a very limited number of Blimp1-mVenus-positive cells overlap with Ki67, suggesting that even if Blimp1 begins expression in mitotic cells, it is likely to be expressed from the last step of the cell cycle.

Functional role of Blimp1 in photoreceptor development We demonstrated that lack of Blimp1 results in decreased numbers of both cone and rod photoreceptor cells. Moreover, Q-PCR and microarray analysis of the control and the Blimp1 CKO retinas also showed that expression of many photoreceptor-related genes except Otx2 were downregulated in the Blimp1 CKO retina. These observations suggest that although initial photoreceptor cell fate is determined by Otx2, approximately half of photoreceptor precursors did not develop normally in the Blimp1 CKO retina. On the other hand, we found that both Chx10-positive bipolar-like cells and proliferation marker-positive cells were notably increased around at P6, and at the same time, cell death was enhanced in the Blimp1 CKO retina.

How does the lack of Blimp1 in photoreceptor precursors induce the overproduction of bipolar-like cells? Several lines of evidence suggest that Blimp1 ablation results in a direct cell fate change from photoreceptor precursors to bipolar-like cells. We demonstrated by reporter assay that Blimp1 can bind to Chx10 enhancer regions and repress Chx10 expression. Moreover, in vivo electroporation of Blimp1 also presented the possibility that Blimp1 blocks bipolar cell development. Intriguingly, the $\mathrm{Cr} x$ LacZ tracer Blimp1 CKO mouse (Blimp1 $1^{\text {flox/flox }} ; \mathrm{Dkk}-\mathrm{Cre}^{+}$; Crx$\mathrm{LacZ}^{+}$) showed that the $\beta$-gal signal partially merged with Chx10, suggesting that Crx-positive postmitotic photoreceptor precursors aberrantly expressed Chx10. We showed previously that strong Otx2 expression is shifted from photoreceptors to bipolar cells after birth (Koike et al., 2007). It was speculated that Otx2 expressed in bipolar cells could promote bipolar cell fate determination by increasing Chx10 expression (Kim et al., 2008). In normal retinal development, Chx10 expression is lost in postmitotic cells, except for bipolar cells and a subset of Müller glia (Rowan and Cepko, 2004). However, in the Blimp1 CKO retina, some photoreceptor precursors that originally express Otx2 may aberrantly upregulate $C h \times 10$ through escaping suppression by Blimp1, and therefore these cells are driven to become bipolarlike cells. Since these cells have already executed a program toward becoming photoreceptors, many of these cells may differentiate into neither photoreceptors nor bipolar cells, and subsequently they are eliminated by apoptosis. How does the loss of Blimp1 in photoreceptor precursors cause an aberrant increase of proliferating cells? We found that PCNA-positive proliferating cells increased in the Blimp1 CKO retina. We also observed that proliferating cells were ectopically localized in the OPL region in the Blimp1 CKO retina at P6. Indeed, Blimp1 is known to regulate proliferation in several tissues by repressing expression of cellcycle regulators (Lin et al., 1997, 2000; Horsley et al., 2006). Blimp1 might function to ensure cell cycle exit and/or prevent newly generated photoreceptor precursor cells from reentering the cell cycle. It has been reported previously that ectopic expression of cyclin D1 or E2F1 in rods results in the ectopic increase of proliferating cells and cell death (Howes et al., 1994; Lin et al., 2001). Furthermore, in the $\mathrm{Rb}^{-/-} ; \mathrm{p107^{+/- }} ; \mathrm{p} 130^{-/-}$retina, even mature horizontal cells were shown to reenter the cell cycle (Ajioka et al., 2007). Some of these cell cycle reentry cells exit the cell cycle again later in development and differentiate either into photoreceptors or bipolar cells, both of which express Otx2. At 
this time point, differentiating progenitors tend to become bipolar cells at a higher rate than they become photoreceptors, since later progenitors produce more bipolar cells than photoreceptors during postnatal retinal development. Given that Otx2 expression begins in the late $\mathrm{S}$ to early G2 phase of the last cell cycle in retinal progenitor cells (Trimarchi et al., 2008a,b), and that a small population of retinal progenitor cells may also express Blimp1 just after Otx2 expression in mitotic progenitors, there is a possibility that Blimp1 deficiency lead to an overproliferation of progenitor cells rather than reentry into the cell cycle. In addition, we observed an increase of $\beta$-gal and Ki67 double-positive cells in the Crx-LacZ tracer Blimp1 CKO mice. This result also supports the idea that excess proliferating cells in the Blimp1 CKO are derived from aberrant photoreceptor precursors.

Again, the possible role of Blimp1 in the repression of Chx10 may account for the mechanism of overproduction of proliferating cells in the Blimp1 $\mathrm{CKO}$ retina. In the embryonic retina, Chx10 is essential for progenitor cell proliferation, and a defect of Chx10 in the retinal progenitor cells causes microphthalmia in both mice and humans (Burmeister et al., 1996; Ferda Percin et al., 2000). It is possible that loss of Blimp1 in the photoreceptor precursors causes a failure to repress Chx10 expression, and that aberrant Chx10 expression in the photoreceptor precursors might prevent proper cell cycle exit or cause abnormal cell cycle reentry of photoreceptor precursors.

In the adult CNS, it has been reported that failure of cell-cycle arrest results in the reexpression of various cell cycle proteins such as cyclins, cyclin-dependent kinases, and PCNA in neurons from patients with various neurodegenerative disorders, including Alzheimer's disease, Parkinson's disease, and stroke (Vincent et al., 1996; Nagy et al., 1997; JordanSciutto et al., 2003; Love, 2003). These studies suggest that mature neurons need to continuously suppress their cell cycle. Together with these studies, the phenotypes observed in the current study might contribute to understanding of the mechanism by which postmitotic neurons regulate the cell cycle machinery.

Thus, our results in this study support the hypothesis that in the Blimp1 CKO retina cell fate-committed photoreceptor precursors autonomously change their cell fate to bipolar-like cells in the majority of the cell population, or to proliferating cells in a small number of cells, and
WT retina

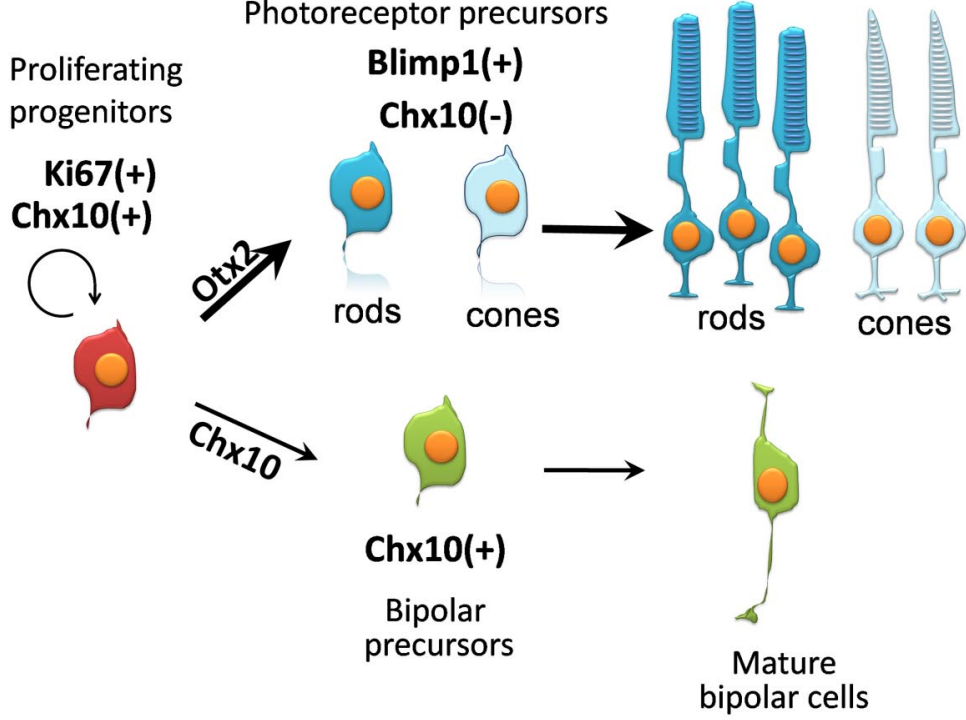

\section{Blimp1 CKO retina}

Blimp1 CKO retina Photoreceptor precursors photoreceptors

Mature

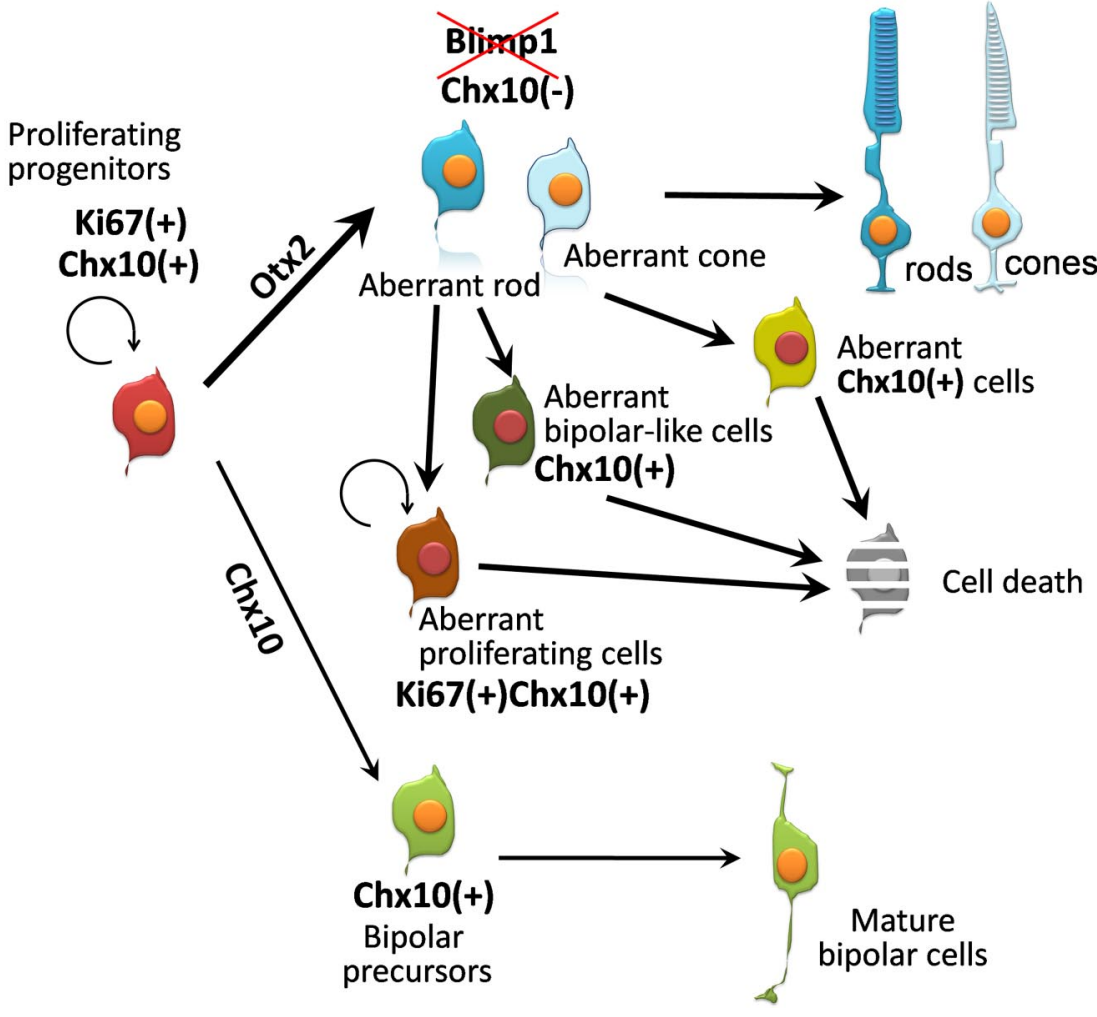

Figure 8. A hypothetical model of the functional mechanism of Blimp1. In this hypothetical model, Blimp1 is expressed in the both rod and cone photoreceptor precursors and ensures photoreceptor cell fate in the wild-type (WT) developing retina (top). In the Blimp 1 CKO retina (bottom), loss of Blimp 1 produces some proportion of rod photoreceptor precursors aberrantly expressing Chx10, resulting in overproduction of bipolar-like cells and proliferating retinal cells. Some proportion of aberrant rod photoreceptors appear to undergo cell fate switch to become bipolar-like cells. The improper cell cycle exit and/or reentry of aberrant photoreceptor precursors into the cell cycle might contribute to the increase of proliferating cells in the Blimp1 CKO retina. The excess bipolar-like cells and proliferating cells derived from abnormal photoreceptor precursors are eliminated by apoptosis. Some proportion of cone precursors in the Blimp1-null retina aberrantly express Chx10 and die at embryonic stages. 
then these abnormal cells die by apoptosis. Blimp1 seems to ensure the cell fate determination of photoreceptor precursors and prohibit aberrant cell fate choice or abnormal cell cycle regulation in the developing retina (Fig. 8).

In the present study, we showed that Blimp1 plays an essential role in photoreceptor development and identified Chx10 as one of the possible targets of Blimp1. However, approximately half of the normal number photoreceptor cells were generated in the Blimp1 CKO retina. One possibility is that another family member or unknown factor compensated for the loss of Blimp1, although we did not detect any significant upregulation of other Prdm family members in the Blimp1 CKO retina in our microarray analysis of the control and the Blimp1 CKO retinas. It should be noted that remaining photoreceptors expressed rhodopsin or cone opsins; however, their outer segments were significantly shorter than those of the control photoreceptors.

Very recently, Brzezinski et al. (2010) independently reported retinal phenotypes in Blimp1 CKO mice using an $\alpha$ Pax6-Cre line, which expresses Cre in retinal progenitors in the peripheral part of the retina. Consistent with our results, they showed an increase of bipolar-marker positive cells instead of a decrease of photoreceptor precursors in the Blimp1 CKO retina. In contrast to our result, they did not mention the increase of proliferating cells in the developing Blimp1 CKO retina. They mentioned excess Sox9positive Müller glial cells in the Blimp1 CKO retina. We also found increased Sox9-positive cells in the Blimp1 CKO; however, these cells at least partially colocalized with the cell proliferation marker, suggesting that Sox9-positive cells are progenitor-like cells rather than Müller glial cells (supplemental Fig. S6, available at www.jneurosci.org as supplemental material). This may require additional analysis in future.

It has been reported that Blimp1 functions with histone methyltransferases (G9a, Prmt5), histone demethylase (LSD1), histone deacetylases, and transcriptional cofactors (Groucho family protein) by recruiting its multiple domains in a contextdependent manner (Ren et al., 1999; Yu et al., 2000; Gyory et al., 2004; Ancelin et al., 2006; Su et al., 2009). Whether or not epigenetical regulation by Blimp 1 plays a role in retinal photoreceptor development awaits future analysis.

\section{References}

Ajioka I, Martins RA, Bayazitov IT, Donovan S, Johnson DA, Frase S, Cicero SA, Boyd K, Zakharenko SS, Dyer MA (2007) Differentiated horizontal interneurons clonally expand to form metastatic retinoblastoma in mice. Cell 131:378-390.

Ancelin K, Lange UC, Hajkova P, Schneider R, Bannister AJ, Kouzarides T, Surani MA (2006) Blimpl associates with Prmt5 and directs histone arginine methylation in mouse germ cells. Nat Cell Biol 8:623-630.

Brzezinski JA, Lamba DA, Reh TA (2010) Blimp1 controls photoreceptor versus bipolar cell fate choice during retinal development. Development 137:619-629.

Burmeister M, Novak J, Liang MY, Basu S, Ploder L, Hawes NL, Vidgen D, Hoover F, Goldman D, Kalnins VI, Roderick TH, Taylor BA, Hankin MH, McInnes RR (1996) Ocular retardation mouse caused by Chx10 homeobox null allele: impaired retinal progenitor proliferation and bipolar cell differentiation. Nat Genet 12:376-384.

Carter-Dawson LD, LaVail MM (1979) Rods and cones in the mouse retina. I. Structural analysis using light and electron microscopy. J Comp Neurol 188:245-262.

Cepko CL (1996) The patterning and onset of opsin expression in vertebrate retinae. Curr Opin Neurobiol 6:542-546.

Cepko CL, Austin CP, Yang X, Alexiades M, Ezzeddine D (1996) Cell fate determination in the vertebrate retina. Proc Natl Acad Sci USA 93:589-595.

Chang DH, Cattoretti G, Calame KL (2002) The dynamic expression pattern of B lymphocyte induced maturation protein-1 (Blimp-1) during mouse embryonic development. Mech Dev 117:305-309.
Ferda Percin E, Ploder LA, Yu JJ, Arici K, Horsford DJ, Rutherford A, Bapat B, Cox DW, Duncan AM, Kalnins VI, Kocak-Altintas A, Sowden JC, Traboulsi E, Sarfarazi M, McInnes RR (2000) Human microphthalmia associated with mutations in the retinal homeobox gene CHX10. Nat Genet 25:397-401.

Furukawa A, Koike C, Lippincott P, Cepko CL, Furukawa T (2002) The mouse Crx 5' -upstream transgene sequence directs cell-specific and developmentally regulated expression in retinal photoreceptor cells. J Neurosci 22:1640-1647.

Furukawa T, Morrow EM, Cepko CL (1997) Crx, a novel otx-like homeobox gene, shows photoreceptor-specific expression and regulates photoreceptor differentiation. Cell 91:531-541.

Furukawa T, Morrow EM, Li T, Davis FC, Cepko CL (1999) Retinopathy and attenuated circadian entrainment in Crx-deficient mice. Nat Genet 23:466-470.

Gyory I, Wu J, Fejer G, Seto E, Wright KL (2004) PRDI-BF1 recruits the histone $\mathrm{H} 3$ methyltransferase G9a in transcriptional silencing. Nat Immunol 5:299-308.

Horsley V, O'Carroll D, Tooze R, Ohinata Y, Saitou M, Obukhanych T, Nussenzweig M, Tarakhovsky A, Fuchs E (2006) Blimpl defines a progenitor population that governs cellular input to the sebaceous gland. Cell 126:597-609.

Howes KA, Ransom N, Papermaster DS, Lasudry JG, Albert DM, Windle JJ (1994) Apoptosis or retinoblastoma: alternative fates of photoreceptors expressing the HPV-16 E7 gene in the presence or absence of p53. Genes Dev 8:1300-1310.

Hsiau TH, Diaconu C, Myers CA, Lee J, Cepko CL, Corbo JC (2007) The cis-regulatory logic of the mammalian photoreceptor transcriptional network. PLoS One 2:e643.

Jordan-Sciutto KL, Dorsey R, Chalovich EM, Hammond RR, Achim CL (2003) Expression patterns of retinoblastoma protein in Parkinson disease. J Neuropathol Exp Neurol 62:68-74.

Kallies A, Nutt SL (2007) Terminal differentiation of lymphocytes depends on Blimp-1. Curr Opin Immunol 19:156-162.

Kallies A, Hawkins ED, Belz GT, Metcalf D, Hommel M, Corcoran LM, Hodgkin PD, Nutt SL (2006) Transcriptional repressor Blimp-1 is essential for $\mathrm{T}$ cell homeostasis and self-tolerance. Nat Immunol 7:466-474.

Keller AD, Maniatis T (1991) Identification and characterization of a novel repressor of beta-interferon gene expression. Genes Dev 5:868-879.

Kim DS, Matsuda T, Cepko CL (2008) A core paired-type and POU homeodomain-containing transcription factor program drives retinal bipolar cell gene expression. J Neurosci 28:7748-7764.

Koike C, Nishida A, Ueno S, Saito H, Sanuki R, Sato S, Furukawa A, Aizawa S, Matsuo I, Suzuki N, Kondo M, Furukawa T (2007) Functional roles of Otx2 transcription factor in postnatal mouse retinal development. Mol Cell Biol 27:8318-8329.

Koyasu T, Kondo M, Miyata K, Ueno S, Miyata T, Nishizawa Y, Terasaki H (2008) Photopic electroretinograms of mGluR6-deficient mice. Curr Eye Res 33:91-99.

Kuo TC, Calame KL (2004) B lymphocyte-induced maturation protein (Blimp)-1, IFN regulatory factor (IRF)-1, and IRF-2 can bind to the same regulatory sites. J Immunol 173:5556-5563.

Lin KI, Lin Y, Calame K (2000) Repression of c-myc is necessary but not sufficient for terminal differentiation of B lymphocytes in vitro. Mol Cell Biol 20:8684-8695.

Lin SC, Skapek SX, Papermaster DS, Hankin M, Lee EY (2001) The proliferative and apoptotic activities of E2F1 in the mouse retina. Oncogene 20:7073-7084.

Lin Y, Wong K, Calame K (1997) Repression of c-myc transcription by Blimp-1, an inducer of terminal $B$ cell differentiation. Science 276:596-599.

Livne-Bar I, Pacal M, Cheung MC, Hankin M, Trogadis J, Chen D, Dorval KM, Bremner R (2006) Chx10 is required to block photoreceptor differentiation but is dispensable for progenitor proliferation in the postnatal retina. Proc Natl Acad Sci U S A 103:4988-4993.

Love S (2003) Neuronal expression of cell cycle-related proteins after brain ischaemia in man. Neurosci Lett 353:29-32.

Martins GA, Cimmino L, Shapiro-Shelef M, Szabolcs M, Herron A, Magnusdottir E, Calame K (2006) Transcriptional repressor Blimp-1 regulates T cell homeostasis and function. Nat Immunol 7:457-465. 
Matsuda T, Cepko CL (2004) Electroporation and RNA interference in the rodent retina in vivo and in vitro. Proc Natl Acad Sci U S A 101:16-22.

Nagy Z, Esiri MM, Cato AM, Smith AD (1997) Cell cycle markers in the hippocampus in Alzheimer's disease. Acta Neuropathol 94:6-15.

Nishida A, Furukawa A, Koike C, Tano Y, Aizawa S, Matsuo I, Furukawa T (2003) Otx2 homeobox gene controls retinal photoreceptor cell fate and pineal gland development. Nat Neurosci 6:1255-1263.

Ohinata Y, Payer B, O'Carroll D, Ancelin K, Ono Y, Sano M, Barton SC, Obukhanych T, Nussenzweig M, Tarakhovsky A, Saitou M, Surani MA (2005) Blimp1 is a critical determinant of the germ cell lineage in mice. Nature 436:207-213.

Ohinata Y, Sano M, Shigeta M, Yamanaka K, Saitou M (2008) A comprehensive, non-invasive visualization of primordial germ cell development in mice by the Prdm1-mVenus and Dppa3-ECFP double transgenic reporter. Reproduction 136:503-514.

Omori Y, Imai J, Watanabe M, Komatsu T, Suzuki Y, Kataoka K, Watanabe S, Tanigami A, Sugano S (2001) CREB-H: a novel mammalian transcription factor belonging to the CREB/ATF family and functioning via the box-B element with a liver-specific expression. Nucleic Acids Res 29:2154-2162.

Peng GH, Chen S (2005) Chromatin immunoprecipitation identifies photoreceptor transcription factor targets in mouse models of retinal degeneration: new findings and challenges. Vis Neurosci 22:575-586.

Ren B, Chee KJ, Kim TH, Maniatis T (1999) PRDI-BF1/Blimp-1 repression is mediated by corepressors of the Groucho family of proteins. Genes Dev 13:125-137.

Rowan S, Cepko CL (2004) Genetic analysis of the homeodomain transcription factor Chx10 in the retina using a novel multifunctional BAC transgenic mouse reporter. Dev Biol 271:388-402.

Rowan S, Cepko CL (2005) A POU factor binding site upstream of the Chx10 homeobox gene is required for Chx10 expression in subsets of retinal progenitor cells and bipolar cells. Dev Biol 281:240-255.

Roy S, Ng T (2004) Blimp-1 specifies neural crest and sensory neuron progenitors in the zebrafish embryo. Curr Biol 14:1772-1777.

Sato S, Inoue T, Terada K, Matsuo I, Aizawa S, Tano Y, Fujikado T, Furukawa T
(2007) Dkk3-Cre BAC transgenic mouse line: a tool for highly efficient gene deletion in retinal progenitor cells. Genesis 45:502-507.

Shaffer AL, Lin KI, Kuo TC, Yu X, Hurt EM, Rosenwald A, Giltnane JM, Yang L, Zhao H, Calame K, Staudt LM (2002) Blimp-1 orchestrates plasma cell differentiation by extinguishing the mature B cell gene expression program. Immunity 17:51-62.

Shapiro-Shelef M, Calame K (2005) Regulation of plasma-cell development. Nat Rev Immunol 5:230-242.

Shapiro-Shelef M, Lin KI, McHeyzer-Williams LJ, Liao J, McHeyzerWilliams MG, Calame K (2003) Blimp-1 is required for the formation of immunoglobulin secreting plasma cells and pre-plasma memory B cells. Immunity 19:607-620.

Su ST, Ying HY, Chiu YK, Lin FR, Chen MY, Lin KI (2009) Involvement of histone demethylase LSD1 in Blimp-1-mediated gene repression during plasma cell differentiation. Mol Cell Biol 29:1421-1431.

Trimarchi JM, Stadler MB, Cepko CL (2008a) Individual retinal progenitor cells display extensive heterogeneity of gene expression. PLoS One 3:e1588.

Trimarchi JM, Harpavat S, Billings NA, Cepko CL (2008b) Thyroid hormone components are expressed in three sequential waves during development of the chick retina. BMC Dev Biol 8:101.

Turner CA Jr, Mack DH, Davis MM (1994) Blimp-1, a novel zinc fingercontaining protein that can drive the maturation of B lymphocytes into immunoglobulin-secreting cells. Cell 77:297-306.

Vincent I, Rosado M, Davies P (1996) Mitotic mechanisms in Alzheimer's disease? J Cell Biol 132:413-425.

Vincent SD, Dunn NR, Sciammas R, Shapiro-Shalef M, Davis MM, Calame K, Bikoff EK, Robertson EJ (2005) The zinc finger transcriptional repressor Blimp 1/Prdm1 is dispensable for early axis formation but is required for specification of primordial germ cells in the mouse. Development 132:1315-1325.

Wilm TP, Solnica-Krezel L (2005) Essential roles of a zebrafish prdm1/ blimp1 homolog in embryo patterning and organogenesis. Development 132:393-404.

Yu J, Angelin-Duclos C, Greenwood J, Liao J, Calame K (2000) Transcriptional repression by blimp-1 (PRDI-BF1) involves recruitment of histone deacetylase. Mol Cell Biol 20:2592-2603. 